\title{
Immunocontraception par la prévention de l'interaction gamétique
}

\author{
S. HAMAMah, M. JeAN*, P. BarRiÈRE*
}

Unité de Biologie de la Reproduction, Département de Gynéco-Obs, Reproduction humaine, et Médecine foetale, Hôpital Bretonneau, 37044 Tours, ${ }^{*}$ Laboratoire de Biologie de la Reproduction, Département de Gynéco-Obs, Hôpital mère-Enfant, 44035 Nantes

\section{RESUME}

L'inhibition de l'action de molécules antigéniques d'origine spermatique ou ovocytaire impliquées dans le processus de la fécondation devient une cible attractive pour l'élaboration de vaccin contraceptif efficace, spécifique et réversible pour les pays en voie de dévéloppement.

Des approches variées à but contraceptif ont été utilisées pour identifier à partir de spermatozoïdes des antigènes impliqués dans l'interaction gamétique et biologiquement importants. Mais, les travaux les plus avancés sur l'effet immunocontraceptif ne concernent que peu d'antigènes spermatiques comme candidats potentiels. En effet, parmi les antigènes du gamète mâle qui ont été identifiés on trouve l'antigène-1 (FA-1) de nature glycoprotéique, isolé du testicule humain, inhibant la fixation des spermatozoïdes sur la ZP. Mais un tel antigène n'est pas spécifique d'espèce. Chez le lapin, une famille antigénique RSA-1, 2, 3 dont les anticorps anti RSA sont capables d'inhiber la fusion entre la membrane plasmique du spermatozoïde (région post-acrosomique) et la membrane plasmique de l'ovocyte. Au total, la plupart des travaux met en évidence le rôle d'inhibition de la liai- son spermatozoïdes-zone pellucide que jouent les anticorps anti-spermatozoïdes. Cependant, seuls deux antigènes spermatiques HS-63 et SP-10 ont été retenus par l'OMS comme candidats potentiels à la contraception.

Mots clés : immunocontraception, contraception, antigènes, spermatozoïde, zone pellucide.

\section{INTRODUCTION}

La fécondation est le processus essentiel de la reproduction sexuée, elle permet d'initier la formation d'un nouvel être par la fusion de deux cellules germinales : le gamète mâle (spermatozoïde) et le gamète femelle (ovocyte). Pour que la fécondation ait lieu, il faut que les spermatozoïdes reconnaissent la zone pellucide, s'y fixent, qu'ils la pénètrent et qu'il y ait fusion avec la membrane plasmique de l'ovocyte. Les étapes initiales de reconnaissance et d'interaction des gamètes sont capitales dans le déroulement de la fécondation. Car ils font intervenir des molécules situées, d'une part sur la tête du spermatozoïde et, d'autre part, sur la zone pellucide de l'ovocyte.

La détermination de la nature et du rôle des molécules impliquées dans l'interaction des spermatozoides avec la zone pellucide devient depuis quelques années une objectif important pour l'élaboration d'une nouvelle approche en immunocontraception (Figure 1). 
1 - vaccin anti sperme

2 - vaccin anti ovocyte

3 - vaccin anti embryon

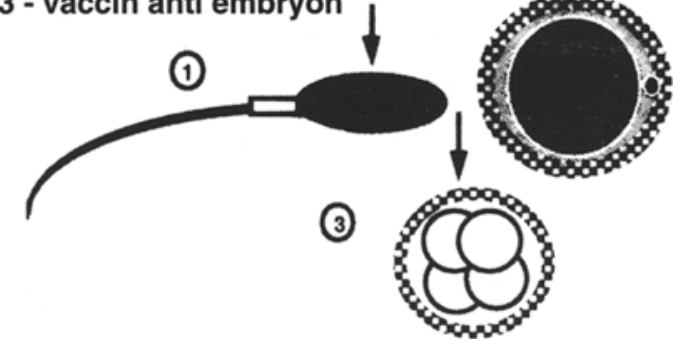

Figure 1 : Cibles pour immunisation contraceptive.

Cependant, afin de comprendre les intérêts de l'interaction gamètique dans la contraception vaccinale, il est indispensable de décrire les cibles potentielles pour l'immunocontraception sur la zone pellucide et le spermatozoïde durant le processus de la fécondation.

\section{RECEPTEURS DE LA ZONE PELLUCIDE}

La zone pellucide, enveloppe acellulaire entourant l'ovocyte des Mammifêres, est synthétisée au cours de la folliculogénèse et assure la protection de l'ovocyte puis de l'embryon jusqu'au stade préimplantatoire. Elle présente une structure élastique et perméable aux enzymes, aux immunoglobulines et aux virus de petite taille.

Les études menées chez la Souris par Bleil et Wassarman [1] ont fourni l'essentiel des informations disponibles actuellement sur la composition biochimique de la zone pellucide. Elle possède une structure tridimensionnelle formée de trois glycoprotéines majeures appelées ZP1, ZP2 et ZP3. La zone pellucide est constituée de longs microfilaments interconnectés et chaque filament est formé par la succession de molécules ZP2 et ZP3 alternées.

La structure de la zone pellucide humaine, désormais bien établie, est formée des 3 glycoprotéines majeures ZP1, ZP2 et ZP3 avec des poids moléculaires apparents res- pectifs de 97,82 et $70 \mathrm{kD}$. Si chez la souris, ZP3 est le seul composant de la zone pellucide capable d'agir comme site de reconnaissance initiale [35], les principaux groupes de glycoprotéines de la ZP humaine auraient tous le rôle de ligands pour les protéines du spermatozoïde humain [26] et pourraient tous déclencher la réaction acrosomique.

Le durcissement de la zone pellucide "hardening" observé après activation de l'ovocyte conduit à une diminution de la solubilité de la zone pellucide et pourrait être interprété comme une augmentation du nombre de ces interactions.

La zone pellucide du fait qu'elle possède des sites antigéniques est done impliquée dans l'interaction avec les spermatozoïdes en assurant la reconnaissance homospécifique et la fixation des spermatozoïdes ainsi que le déclenchement de la réaction acrosomique. Après fécondation, la zone pellucide participe au blocage de la polyspermie et assure la protection de l'embryon au stade préimplantatoire. Les enzymes contenues dans les granules corticaux de l'ovocyte modifient la composition de la zone pellucide. La ZP3 est donc modifiée en ZP3f de poids moléculaire identique mais inapte à fixer de nouveau le spermatozoïde. La ZP2 $(120 \mathrm{kD})$ est clivée sous l'action d'une ZP2-protéinase en $\mathrm{ZPf}(93 \mathrm{kD})$ [15].

L'interaction spermatozoïdes-zone pellucide, à l'image des interactions entre cellules somatiques, serait du type ligand-récepteur [9]. Selon le modèle proposé chez la Souris par Bleil et Wassarman, l'interaction du spermatozoïde avec la zone pellucide peut être séparée en 2 étapes: la première étape débute par une liaison labile et réversible du spermatozoïde avec la zone pellucide. Puis, l'interaction des chaînes oligosaccharidiques de ZP3 avec un (ou plusieurs) récepteurs de la membrane plasmique du spermatozoïde établit une liaison irréversible et spécifique entre les gamètes. 


\section{RECEPTEURS DE SPERMATOZOÏDE}

Chez les mammifêres, certaines protéines et glycoprotéines présentes sur la membrane plasmique de spermatozoïde, impliquées dans la reconnaissance et la fixation de spermatozoïdes sur la zone pellucide ont été caractérisées. Si la nature et la localisation des récepteurs spermatiques impliqués dans l'interaction avec la zone pellucide ne sont pas encore totalement précisées, le rôle des protéines de la membrane plasmique du spermatozoïde a cependant pu être établi dans plusieurs espèces.

Chez la souris, une galactosyltransférase portée par la membrane plasmique du spermatozoïde pourrait être impliquée dans la reconnaissance et la fixation des spermatozoïdes $[13,14]$. La liaison avec la zone pellucide résulterait donc d'un pontage entre la membrane plasmique du spermatozoïde et un résidu $\mathrm{N}$-acétylglucosamine de la ZP3 par l'intermédiaire de cette enzyme.

Chez l'homme, il n'y aurait pas de galactosyltransférase mais, une $\alpha$-D-mannosidase, retrouvée à la surface du spermatozoïde qui serait le récepteur pour ZP3[34]. Grâce à des anticorps anti-phosphotyrosine (anti PTyr), des protéines servant de substrat à des protéines-kinases ont également pu être identifiées dans les spermatozoüdes murins et humains.

Après capacitation, $15 \%$ des spermatozoïdes murins et $50 \%$ des spermatozoides humains possèdent des résidus P-Tyr au niveau de la région acrosomique. Chez la souris, l'incubation de spermatozoïdes capacités avec des pellucides dissoutes fait apparaître une réactivité importante à l'anticorps anti PTyr pour une protéine de $95 \mathrm{kD}$, appelée $\mathrm{P}$ 95. Chez l'homme, la phosphorylation sur tyrosine d'une protéine de $94 \mathrm{kD}$ est également stimulée par l'exposition à des pellucides dissoutes. Ainsi, à l'image de la souris où la P95 se fixe à ZP3, la P 94 du spermatozoïde humain pourrait être impliquée dans l'interaction avec la zone pellucide.
L'analogie retrouvée entre 3 protéines du spermatozoïde humain $(16,18$ et $19 \mathrm{kD})$ et la RSA "Rabbit Sperm Antigen" qui interagit de façon spécifique avec la zone pellucide, est également en faveur du rôle de ces protéines comme récepteurs pour la $\mathrm{ZP}$ chez l'Homme [18].

Deux modalités d'interactions entre les spermatozoïdes et la zone pellucide ont pu être observées :

- chez la plupart des mammiferres, la fixation des spermatozoïdes n'a lieu que si la membrane plasmique est intacte, il en est de même chez l'homme,

- chez le hamster, la fixation du spermatozoïde sur la zone pellucide ne peut s'effectuer qu'après la réaction acrosomique. Une fois fixé à la zone pellucide (fixation primaire) le spermatozoïde effectue sa réaction acrosomique. Ce processus d'exocytose assure d'une part, la libération du contenu acrosomique et, d'autre part, l'externalisation de la membrane interne de l'acrosome [37]. L'acrosine est une protéase localisée dans l'acrosome des spermatozoüdes de mammifères sous sa forme inactive, la proacrosine. Cette enzyme pourrait être impliquée dans la fixation secondaire qui intervient entre le spermatozoïde en cours de réaction acrosomique et les chaînes saccharidiques de ZP2.

Chez la souris, après l'étape de fixation primaire du spermatozoïde, ZP3 est inactivée en ZP3f [28] perdant ainsi sa capacité à fixer de nouveaux spermatozoïdes. Après la fixation secondaire, la protéolyse de ZP2, qui empêche la pénétration de spermatozoïdes surnuméraires, complète le processus de blocage de la polyspermie. Ces modifications de ZP2 et ZP3, observées au cours de la fécondation de l'ovocyte de souris, sont provoquées par la libération dans l'espace périvitellin des enzymes des granules corticaux. Chez l'homme, le blocage de la polyspermie pourrait être associé à une dégradation de ZP1. 
Le mécanisme et la nature chimique selon lesquels les récepteurs de la membrane plasmique de la tête des spermatozoïdes reconnaissent ceux de la zone pellucide et s'y fixent commence maintenant à être élucidés dans certaines espèces (Figure 1, Tableau 1).

\section{IMMUNOCONTRACEPTION PAR LA PREVENTION DE L'INTERACTION GAMETIQUE}

\section{Anticorps antispermatozoïdes}

L'idée d'obtenir un vaccin dirigé contre les spermatozoïdes a été suggèrée il y a plus de 90 ans par Metchnikoff.

Le spermatozoïde est une cellule fortement antigénique, mais la rareté de la détection d'anticorps anti-spermatozoïdes chez l'homme implique un système de tolérance de l'individu à l'encontre de ses spermatozoïdes. En effet, les antigènes spermatiques apparaissent tardivement dans le développement de l'individu. A la puberté il apparaît lors du début de la spermatogénèse de nouveaux antigènes sur la surface des spermatozoïdes.
Selon les études, approximativement 5\% des hommes infertiles ont des anticorps dirigés contre les spermatozoïdes. Dans un but d'obtenir un vaccin contraceptif, des anticorps contre certaines protéines de spermatozoïdes ont été aussi identifiées. L'aspect hétérogène des anticorps antispermatozoïdes laisse entrevoir la complexité antigénique du spermatozoïde. La membrane plasmique des spermatozoïdes de mammifêres est une véritable mosaique, regroupant des surfaces limitées bien localisées sur le spermatozoïde, presentant une spécificité d'espèce, et possédant des propriétés intra-membranaires constituant ainsi des récepteurs spécifiques.

La plupart de ces anticorps anti-spermatozoïdes agissent à une ou plusieurs étapes de la fécondation. Mais, quel que soit le vaccin contraceptif obtenu à partir de protéines de spermatozoïdes, les protéines doivent repondre à des exigences telles que :

- définir les protéines spermatiques pouvant être utilisés comme immunogènes,

- être présents sur la surface des spermatozoides.

Tableau 1 : Molécules impliquées dans la fuxation des spermatozoïdes sur la zone pellucide.

\begin{tabular}{|c|c|c|c|}
\hline Espèces & Spermatozoïde & Zone Pellucide & Réferences \\
\hline \multirow[t]{3}{*}{ Souris } & Protéine SP 56 & Galactose & {$[2]$} \\
\hline & $\begin{array}{l}\beta-1,4 \text { Galactosyltransférase } \\
\text { (GalTase) }\end{array}$ & N-Acétylglucosamine* & [14] \\
\hline & Protéine (P 95) & ZP3 & {$[12]$} \\
\hline Rat & $\alpha$-D-Mannosidase & " $\alpha$-Linked" D-Mannose* & {$[34]$} \\
\hline Porc & Proacrosine & Fucoidine-like & [8] \\
\hline Lapin & Protéines RSA & non spécifique & [18] \\
\hline Cobaye & Protéine PH-20 & non spécifique & {$[20]$} \\
\hline Homme & $\begin{array}{l}\text { Proacrosine } \\
\alpha \text {-D-Mannosidase }\end{array}$ & $\begin{array}{l}\text { Fucoidine-like } \\
\text { " } \alpha \text {-Linked" D-Mannose* }\end{array}$ & {$[8]$} \\
\hline
\end{tabular}

* Fixation de type Enzyme-Substrat 
Des approches variées à but contraceptif ont été utilisées pour identifier à partir de spermatozoïdes des antigènes impliqués dans l'interaction gamétique et biologiquement importants. Mais, les travaux les plus avancés à effet immunocontraceptif ne concernent que peu d'antigènes spermatiques comme candidats potentiels. En effet, parmi les antigènes du gamète mâle qui ont été identifiés on trouve l'antigène-1 (FA-1) de nature glycoprotéique, isolé du testicule humain, inhibant la fixation des spermatozoïdes sur la zone pellucide. Mais un tel antigène n'est pas spécifique d'espèce. Chez le lapin, O'Rand et al [17] ont identifié une famille antigénique RSA-1, 2, 3 dont les anticorps anti RSA sont capables d'inhiber la fusion entre la membrane plasmique de spermatozoïde (region post-acrosomique) et la membrane plasmique de l'ovocyte. L'immunisation active de souris avec la lactate déshydrogénase spécifique des spermatozoïdes (LDH-C4) a permis d'inhiber la fertilité des mâles, mais des réactions indésirables (orchite) chez certains mâles ont été observées. Au total, la plupart des travaux met en évidence le rôle d'inhibition de la liaison spermatozoïdeszone pellucide que jouent les anticorps antispermatozoïdes. Cependant, seuls deux antigènes spermatiques HS-63 et SP-10 ont été retenus par l'OMS comme candidats potentiels à la contraception.

\section{Anticorps anti-zone pellucide}

Du fait des rôles majeurs de la zone pellucide dans le processus de la fécondation (interaction avec les spermatozoïdes, induction de la réaction acrosomique et contrôle de la polyspermie), la zone pellucide est également la cible attractive pour le développemnt d'un vaccin contraceptif. Elle présente une forte immunogénicité hétérospécifique [27] et les anticorps obtenus inhibent fortement la fécondation in vitro [23]. Des analyses immunologiques ont révélé la présence au sein de la zone pellucide d'anti-

Table 2. Candidats potentiels à la contraception d'origine spermatique

\begin{tabular}{|c|c|c|c|c|}
\hline Espèce & Antigènes & $\begin{array}{l}\text { Localisation sur le } \\
\text { spermatozoïde }\end{array}$ & Fonction ciblée & Réf. \\
\hline Lapin & RSA $1,2,3$ & membrane plasmique & fusion spermatozoïde/ovocyte & [17] \\
\hline Homme & FA-1 & $\begin{array}{l}\text { membrane plasmique } \\
\text { de la cellule germinale }\end{array}$ & fixation spermatozoïdes/ZP & {$[16]$} \\
\hline Homme & HS-63 & protéine acrosomique & réaction acrosomique & [11] \\
\hline $\begin{array}{l}\text { Homme, } \\
\text { Babouin }\end{array}$ & SP10 & $\begin{array}{l}\text { membrane acrosomique } \\
\text { interne }\end{array}$ & fusion spermatozoïde/ovocyte & {$[7]$} \\
\hline Homme & SP 56 & membrane plasmique & fixation spermatozoïdes/ZP & {$[2]$} \\
\hline Cobaye & $\begin{array}{l}\text { PH-20 } \\
\text { PH-30 }\end{array}$ & $\begin{array}{l}\text { membrane plasmique } \\
\text { membrane plasmique }\end{array}$ & $\begin{array}{l}\text { fixation spermatozoïdes/ZP } \\
\text { fusion spermatozoide/ovocyte }\end{array}$ & {$[21]$} \\
\hline Porc & AWN-1, 2 & membrane plasmique & fixation spermatozoïdes/ZP & {$[25]$} \\
\hline Souris & $\begin{array}{l}\text { Galactosyl } \\
\text { transférase }\end{array}$ & $\begin{array}{l}\text { membrane plasmique } \\
\text { (région péri-acrosomique) }\end{array}$ & fixation spermatozoïdes/ZP & {$[30]$} \\
\hline
\end{tabular}


gènes spécifiques d'espèce impliqués dans la reconnaissance de spermatozoïdes pour la zone pellucide, ayant des réactions croisées avec d'autres espèces de mammifères [4].

Les antigènes de la zone pellucide répondent à une partie des exigences requises pour constituer la cible d'anticorps "contraceptifs". Ces antigènes sont hautement immunogènes, au moins en ce qui concerne l'hétéroimmunisation, mais peu immunogènes en isoimmunisation. Plusieurs travaux ont montré la présence d'anticorps anti-zona dans le sérum de femmes fertiles $[28,3]$. Les antisera dirigés contre les composants de la zone pellucide ne sont pas strictement spécifiques d'espèce, puisqu'il existe des réactions croisées entre de nombreux mammifêres (Tableau 3).

L'inconvénient majeur de l'utilisation d'anticorps anti- zone pellucide à but contracep- tif, est l'apparition d'effets secondaires, notamment dans le protocole d'hétéroimmunisation. Ainsi chez le lapin immunisé par la zone pellucide de porc, l'effet contraceptif est intense mais s'accompagne de troubles ovariens [36]. De tels effets indésirables ne sont pas surprenants puisque les antigènes visés sont aussi portés par les ovocytes de la réserve ovarienne.

Il reste encore de nombreux aspects à éclaircir concernant la structure antigénique du spermatozoïde et de la zone pellucide avant d'espérer l'obtention d'un moyen contraceptif capable d'inhiber seul l'interaction spermatozoïdes-zone pellucide et de permettre leur utilisation dans la contraception participant ainsi à la maîtrise et à la régulation de la démographie mondiale.

\section{Tableau 3 : Réaction des anticorps anti zone pellucide.}

\begin{tabular}{llc}
\hline $\begin{array}{l}\text { Anticorps } \\
\text { anti zone pellucide }\end{array}$ & $\begin{array}{l}\text { Réaction croisée } \\
\text { avec zone pellucide de }\end{array}$ & Références \\
\hline Hamster & $\begin{array}{l}\text { Souris } \\
\text { Souris, Singes rhésus } \\
\text { Ecureuil }\end{array}$ & {$[31]$} \\
& Souris, Hamster & {$[5]$} \\
Rat & Rat, Hamster & $(32)$ \\
Souris & Porc & $(33)$ \\
Lapin & Lapin, Singes rhésus & $(36)$ \\
& Marmoset, Chien, & \\
Bovin & Humain, Hamster & {$[6]$} \\
& Chimpanzé, ouistiti & \\
Porc & Lapin, Humain, Singe, écureuil & {$[29]$} \\
& Lapin & {$[24]$} \\
& Humain & {$[36]$} \\
& Humain, ouistiti & {$[10]$} \\
Humain & Porc & {$[19]$} \\
& Porc, ouistiti, Chimpanzé & {$[22]$} \\
\hline
\end{tabular}




\section{REFERENCES}

1. BLEII. JD, WASSARMAN PM : Structure and function of the zona pellucida : identification and characterization of the proteins of the mouse oocyte's zona pellucida. Dev. Biol $1980 ; 76: 185-203$.

2. BLEIL JD, WASSARMAN PM : Identificate of ZP3-binding protein on acrosome intact mouse sperm by photoaffinity crosslinking. Proc; Nat. Acad. Sci (USA) $1990 ; 87: 5563-5567$.

3. CAudle MR, Shivers CA, Wil, RA : Clinical significance of naturally occuring anti-zona pellucida antibodies. Am. J. Reprod. Immunol. Microbio $1987 ; 15: 119-121$.

4. Dreli, DW, Dunbar BS : Monoclonal antibodies to rabbit and pig zonae pellucidae distinguish species-specific and shared antigenic determinants. Biol. Reprod 1984 ; 30 : 445-457.

5. GWatKin RBL, Wilitams DT, Carlo DJ : Immunization of mice with heat-solubilized hamster zonae: production of anti-zona antibody and inhibition of fertility. Fertil. Steril $1977 ; 28: 871$ 877.

6. Gwatkin RBI, Williams DT, Meyenhofer M. : Isolation of bovine zona pellucida from ovaries with collagenase: antigenic and sperm receptor properties. Gamete Res $1979 ; 2: 187-194$.

7. Herr JC, Wright RM, John E, Foster J, Kays T, FLICKINGER CJ : Identification of human acrosomal antigen SP-10 in primates and pigs. Biol. Reprod $1990 ; 42: 377-382$.

8. JoNes R, BRown CR, LANCASTER RT : Carbohydrate binding properties of boar sperm proacrosin and assessment of its role in sperm-egg recognition and adhesion during fertilization. Development 1988 ; $1.02: 781-792$.

9. Kopf GS, Wilise MW : Signal transduction processes leading to acrosomal exocytosis in Mammalian spermatozoa. TEM September/October. Elsevier, Science, Publishing Co, 1990 ; 362-368.

10. Koyama $K$, Hasegawa A, Inole $M$, Isojima $S$ : Blocking of human-sperm zona interaction by monoclonal antibodies to a glycoprotein family (ZP4) of porcine zona pellucida. Biol. Reprod 1991 ; 45 : 727-7345.

11. LEE SL, WEI YH : The involvment of extracellular proteinases and proteinases inhibitors in mammalian fertilization. Biotechnol. Appled. Biochem $1984 ; 19: 31-41$.

12. Leytox $\mathrm{L}$, SALING PM : $95 \mathrm{kD}$ sperm proteins bind ZP3 and serve as tyrosine kinase substrates in response to zona binding. Cell $1989 ; 57: 1123-$ 1130 .
13. Lopez LC, Bayna EM, Litory D, Sinher NL, ShaPER JH, SHUR BD : Receptor function of mouse sperm surface galactosyl-transferase during fertilization. J. Cell. Biol 1985 ; 101 : 1501-1510.

14. МACEK MB, SHLR BD : Protein carbohydrate complementary in mammalian gamete recognition. Gamete. Res 1988 ; 20 : 93-109.

15. Molier CC, WASSARMANN PM : Characterization of a proteinase that cleaves zona pellucida glycoprotein ZP2 following activation of mouse eggs. Dev. Biol 1989 ; 132 : 103-112.

16. NAZ RK, AIEXANDER NJ, ISAHAKIA M : Monoclonal antibody to a human germ cell membrane glycoprotein that inhibts fertilization. Science $1984 ; 22: 342-344$.

17. O'Rano MG, MatThews JE, Welch JE, Fisher SJ : Identification of zona binding proteins of rabbit, pig, human and mouse spermatozoa on nitrocellulose blots. J. Exp. Zool $1985 ; 235: 423-428$.

18. O'RAND MG, WIDGREEN EE, NIKOIAJCZYK BS, RICHARDSON RT, SHABANOWITZ RB : Receptors for zona pellucida on human spermatozoa, In : Gamete interaction : Prospects for immunocontraception, Wiley-Liss Inc $1990 ; 213-224$.

19. Patersox M, Koothax P'T, Morris KD, O'Byrnf KT, Braude P, Williams A, AitKen RJ : Analysis of the contraceptive potential of antibodies against native and degly cosylated porcine ZP3 in vivo and in vitro. Biol. Reprod $1992 ; 46: 523-534$.

20. Primakofk P, Lathrop W, Woolman L, Coway A, MYLES DG : Fully effective contraception in male and female guinea pigs immunized with the sperm protein PH-20. Nature $1988 ; 335: 543-656$.

21. Primakoff P, Myles DG : Selection of appropriate sperm antigens and animal models for developing an anti-sperm contraceptive vaccine. In : Bacetti B (ed). Comparative Spermatology. 20 years after. NY : Raven Press $1992 ; 887-892$.

22. SACCO AG : Antigenic cross-reactivity between human and pig zona pellucida. Biol. Reprod $1977 ; 16: 164-168$.

23. SACCO AG : Inhibition of fertility in mice by passive immunization with antibodies to isolated zonae pellucidae. J. Reprod. Fert 1979 ; 56 : 533-537.

24. Sacco AG, Yurewicz EC, Slbramaniax MG, De MaYo FJ : Zona pellucida composition: species cross-reactivity and contraceptive potential of antiserum to a purified pig zona antigen (PPZA). Biol. Reprod 1981 ; 25 : 997-1108.

25. Sanz L, Calvete JJ, Mann K, Schafer W, Schmid ER, AmShlgRUjer W, Sinowat\% F, Ehritard M, TOPER-PETERSON E : The complete primary structure of spermadhesion AWN, a zona pellucida-binding protein isolated from boar spermatozoa. FEBS. Lett. 1992 ; $300: 213-218$. 
26. ShABANowitz RB, O'RAND MG : Characterization of the human zona pellucida from fertilized and unfertilized eggs. J. Reprod. Fertil. 1988 ; 82 : 151161.

27. Shabanowitz RB : Mouse antibodies to human zona pellucida: evidence that human ZP3 is strongly immunogenic and contains two distinct isomer chains. Biol. Reprod1990; $43: 260-270$.

28. Shivers CA, Dusibar BS : Autoantibodies to zona pellucida: a possible cause for infertility in women. Science. $1977 ; 197$ : 1082-1084.

29. Shivers Ca, Gengosian N, Franklin S, MC LAUGHLIN, CA : Antigenic cross-reactivity between human and marmoset zonae pellucidae, a potential target for immunocontraception. J. Med. Primat. $1978 ; 7: 242-248$.

30. Shur BD, NeELy CA : Plasma membrane association, purification, and partial characterization of mouse sperm b 1,4-galactosyltransferase. J. Biol. Chem. $1988 ; 263: 17706-17714$.

31. Tsunoda Y, CHANG MC : In vivo and in vitro fertilization of hamster, rat and mouse eggs for treatment with anti-hamster ovary antiserum. J. Exp. Zool. 1976a; 195 : 409-413.

32. Tsunoda Y, Chang MC : Effect of anti-rat ovary antiserum on the fertilization of rat, mouse and hamstereggs in vivo and in vitro. Biol. Reprod. $1976 \mathrm{~b} ; 14: 354-361$.

33. Tsuxoda Y, Chaig, MC : Effects of antisera on fertilization of mouse, rat and hamster eggs. Biol. Reprod. 1978 ; 18 : 468-474.

34. Tulsinyi DRP, Skudlarek MD, ORgebiN-Crist MC : Human sperm plasma membrane possess alpha-D-mannosidase activity but no galactosyltransferase activity. Biol. Reprod. $1990 ; 42: 843$ 858.
35. Wassarmai PM, Bleit JD, Florman HM, Greve. JM, Rolleir RJ, Salzmann GS : Nature of the mouse egg's receptor for sperm. In: Hedrick JL ed, The molecular and cellular biology of fertilization, New-York, Plenum Press, 1986 ; 55-77.

36. Wood DM, DLnbar BS1 : Direct detection of two cross-reactive antigens between porcine and rabbit zonae pellucidae by radioimmunoassay and immunoelectrophoresis. J. Exp. Zool. 1981 ; 217 : 423433.

37. YANAGIMACHI $R$ : Mammalian fertilization. In : The physiology of reproduction. Knobil E, Neill J eds, New-York, Raven Press I.td, 1994 ; 189-232.

\section{ABSTRACT}

\section{Immunoinhibition of fertilization by contraceptive vaccine}

\author{
S. Hamamah, M. Jean, P. BarRiere
}

The paper summarize the main data relevant to the obtention of contraceptive vaccines based on spermatozoa as well as zona pellucida antigens. The development of novel forms of contraception is one way in which this global population problems can be tackled. The sperm as well as the oocyte antigens are studied as possible contraceptive vaccine candidates are the subject of this review.

Key Words : immunoinhibition of fertilization, contraceptive vaccine, spermatozoon, zona pellucida, antigens. 